\title{
MonoVision-Based Vehicle Detection, Distance and Relative Speed Measurement in Urban Traffic
}

\author{
M. Ibarra Arenado, J. M. Pérez Oria, C. Torre-Ferrero, L. Alonso Rentería
}

Control Engineering Group. Department of Electronic Technology and Systems Engineering, University of Cantabria, Santander, Spain

\begin{abstract}
This paper presents a monovision-based system for on-road vehicle detection and computation of distance and relative speed in urban traffic. Many works have dealt with monovision vehicle detection, but only a few of them provide the distance to the vehicle which is essential for the control of an Intelligent Transportation System (ITS). The system proposed integrates a single camera reducing the monetary cost of stereovision and RADAR-based technologies. The algorithm is divided in three major stages. For vehicle detection we use a combination of two features: the shadow underneath the vehicle and horizontal edges. We propose a new method for shadow thresholding based on the grayscale histogram assessment of a region of interest on the road. In the second and third stages, the vehicle hypothesis verification and the distance are obtained by means of its number plate whose dimensions and shape are standardized in each country. The analysis of consecutive frames is employed to calculate the relative speed of the vehicle detected. Experimental results showed excellent performance in both vehicle and number plate detections and in the distance measurement, in terms of accuracy and robustness in complex traffic scenarios and under different lighting conditions.
\end{abstract}

Index Terms- Driving assistance systems, Intelligent transportation, Monocular vision, Vehicle detection, License plate detection, Distance measurement. 


\section{Introduction}

The automotive industry is constantly evolving as there is a growing interest in the field of safety. Among active safety elements, special mention must be made of Advanced Driving Assistance Systems (ADAS) which alert the driver of a risky situation as soon as possible. If there is no reaction from the driver, these systems, as a last resort, assist in an active way in order to prevent accidents. The work presented in this paper is framed within Forward Collision Avoidance Systems. The goal of them is to aid drivers to maintain a safe stopping distance related to the vehicle in front in order to avoid or at least reduce the number and severity of traffic accidents [1]. Unfortunately, either due to negligence or distraction, the safety distance is not maintained in many cases, especially in cities where most accidents are rear-end collisions. We propose a vision-based system to detect the vehicle ahead in the target path and measure the gap distance and relative speed by means of one single camera. To date, the lack of works dealing with the issue of monovision-based vehicle distance estimation makes this task very challenging [2]. The system proposed is specifically designed to work in urban traffic where the speed limit is $50 \mathrm{Km} / \mathrm{h}$ and most of the front-to-rear collisions take place at low speeds. An important issue to take into account in the development of an active safety system is the economical aspect. Currently, RADAR-based systems are a very popular solution for FCAS, however, due to the sensors' high price, most of them are exclusively aimed at high class vehicles. One of the main targets of the approach proposed here is the development of a low-cost active safety system for a standard road vehicle. Unlike the aforementioned expensive technologies, computer vision is a cheaper technology. The use of a single camera makes the system proposed a low-cost solution suitable for low and medium class vehicles.

The remainder of this paper is organized as follows. Section II reviews the related research. Section III presents an overview of the proposed method, the region of interest establishment, the vehicle detection procedure, the distance-size relationship, the license plate location and the distance and relative speed measurement. Experimental results of measurement accuracy and 
detection reliability are given in Section IV. Finally, Section V summarizes and discusses future work.

\section{Related Work}

Many approaches to assist drivers in avoiding collisions have been developed based on different technologies such as RADAR [3], LASER scanner [4], ultrasounds [5], or vision systems [6-8]. They have benefits and drawbacks. Regarding the latter, RADAR has a narrow field of view and as they are active sensors, they may interfere with other RADAR emitting sources including vehicles with the same technology on board; LASERS are affected by fog and heavy rain; ultrasounds easily fail due to lateral wind; and vision systems can be affected by the changing lighting of outdoor applications and the image processing involves a large computational load which can compromise the real-time performance. Due to these facts, some approaches have focused on a combination of some of the above in order to achieve more reliability [9-11]. However, visual information makes computer vision a versatile technology, able to extract scene information such as road lane, traffic signs and detailed object shape.

The existing vision systems for vehicle detection are based on both mono and stereovision, but regarding the target of vehicle distance measurement, stereovision is currently the only one that can provide accurate depth information [12-14]. The main drawback of stereovision is that it involves finding correspondence between images, which is a complex task that demands a lot of processing time. Monovision has the advantage of a lower image processing time. However, it does not provide depth information, so it is difficult to accurately determine the vehicle's distance. Distance and relative speed parameters are essential for the control of a Forward Collision Avoidance System.

Many monovision-based approaches in the literature deal with vehicle detection but only a few of them estimate the distance. They are very dependent on the assumptions imposed or specific training scenarios that strongly limit the performance. In [15], scene depth is deduced from texture 
gradients, defocus, colour, and haze using a multi-scale Markov Random Field. Images and ground-truth depth maps are used to train the MRF model. The disadvantage is that the use of a finite set of training images implies that the system may not work properly in scenarios that differ from the training set. In [16], the vehicle's distance measurement is based on the contact between the vehicle's tyres and the road. This method could easily overestimate the measure due to the difficulty of getting the exact tire-asphalt contact point which in addition does not correspond to the real back of the vehicle. [17] uses known information about the vehicle appearance (the vehicle's underside) and the distance is determined using the inverse perspective transform assuming flat earth. Finally, [18] assumes an ideal vehicle width for all vehicles and uses the camera pinhole model to determine the distance of the vehicle.

The distance measurement approach proposed in this paper takes advantage of the vehicle's rear number plate. The size and shape of the plate's characters are fixed in each country so the vehicle's distance measurement procedure is simplified to the number plate characters' distance and it can be calculated in a direct and accurate way, free of assumptions. The procedure presented is made up of three separate steps: vehicle detection, number plate localization and finally, distance and relative speed measurement.

Regarding the vehicle detection, most of the monovision approaches developed are based on the search for specific patterns using information about the vehicles' features such as symmetry [19], texture [20], color [21], edges [22], corners [23] and motion features [24]. These approaches have been demonstrated to be effective under a specific environment, but they are very dependent on illumination.

The vehicle detection procedure proposed is based on two features: the shadow underneath the vehicle and horizontal edges. Shadows have already been employed as an indication pattern in $[8,25-27]$. The area underneath the vehicle is darker than any other, regardless of lighting conditions. The shadow's intensity depends on the illumination so the threshold is not fixed. We propose a systematic way to establish an appropriate adaptive threshold that uses the histogram 
associated with only a region of interest on the road corresponding to a safety area of our vehicle's trajectory.

Finally, there are also many approaches to address license plate detection. Most of them work only under restricted conditions such as fixed illumination and stationary backgrounds. They are focused on motionless vehicle applications such as parking management, automatic highway toll collection, etc. The most common approaches are based on filtering strategies and feature matching methods. There are methods based on projective invariance [28], Hough transform [29], color or texture features [30-31], morphology [32], artificial neural networks [33], SVM classifiers to model character appearance variations [34], and methods based on top-hat transform [35-37]. The number plate location approach proposed adapts the widely employed morphological top-hat method to vehicles in motion where the position of the vehicle is unknown and therefore the dimensions of the number plate in the image are, in principle, unknown.

\section{System Description}

\subsection{Method Overview}

In order to measure the distance of an object from a single image it is necessary to have a frontal view and to know the true magnitude of the object. Unfortunately, the dimensions of vehicles are different depending on the make and model, so they cannot be used as a reference. However, a common element on the back of all vehicles is the license plate. It must be approved and its shape and dimensions are fixed in each country. Localizing the front vehicle's number plate and having previously established a relationship between the number plate's size in the image and the distance to the camera, the vehicle's distance can be obtained directly.

After capturing a grayscale frame, the first step consists of establishing of a region of interest on the road corresponding to the safety area in front of our vehicle. Any vehicle circulating inside this safety area is susceptible to a possible rear-end collision. Next, the vehicle detection step begins and a first distance estimation is performed based on the vehicle's bounding box location. Then 
the search of the vehicle's number plate is used for two purposes: to validate the vehicle's detection and to obtain the vehicle's distance. Remember that the relationship between the dimensions of the number plate in the image and the distance to the camera has already been established. Finally, the analysis of consecutive images is employed to obtain the vehicle's relative speed.

\subsection{Region of Interest}

The camera is placed beside the rear-view mirror to capture the scene in front of the vehicle. In addition to the road and vehicles travelling ahead, many other objects can appear in a vehicle's frontal image. A region of interest of the road (ROI) is very important because it simplifies the scene, focusing only on the area risk of a rear-end collision and avoiding the analysis of the part of the road without influence in our trajectory (Fig. 1). In this way, the possibility of errors, false positive detections and computational load are reduced, and the vehicle detection reliability is increased.

The ROI is considered to be comprised of the safety area, that is, the stretch of the road of $10 \mathrm{~m}$ in front of our vehicle with a width equal to our vehicle's width. Only the vehicles within the safety area influence our vehicle's trajectory, therefore only these vehicles are detected by the system, ignoring the useless image portion. The approach proposed is specifically for urban traffic, detecting vehicles ahead in a range up to $10 \mathrm{~m}$ at speeds up to the urban speed limit of $50 \mathrm{Km} / \mathrm{h}$.

\subsection{Vehicle Detection}

The vehicle detection procedure is based on two features: the shadow underneath the vehicle and the lower horizontal edge of this shadow. A distinctive feature of vehicles is the shadow underneath them. Its intensity depends on the illumination, which in turn depends on the weather, but it is always present on the road. Due to the vehicles' morphology the space between the vehicle's underside and the road is small so the road area under the vehicle is not exposed to 


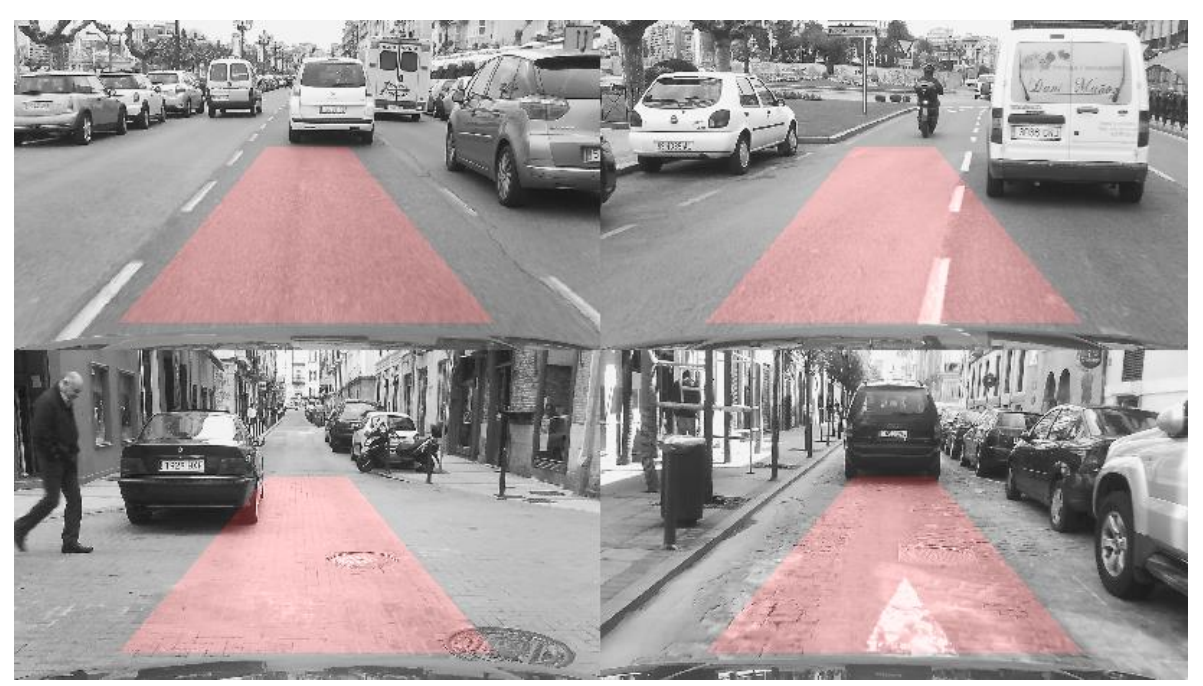

Fig. 1. Vehicle's search area (ROI) corresponding to the safety area.

direct sunlight and it is only affected by a little quantity of lateral diffuse light. This lack of light makes this road area very dark and free of brightness, regardless of lighting conditions, texture and color of the asphalt. Even if the road is shaded, the vehicles' underside is darker than its surroundings. This phenomenon is mathematically explained in [25]. On the other hand, any other element of the road (lateral shadows, potholes, manhole covers, etc) is exposed to both direct and diffuse light which makes it clearer and brighter. Although these elements can be dark they do not exceed the darkness intensity of the shadow under the vehicle [25].

On cloudy days, vehicles are only lit by diffuse light which comes from all directions so it creates little or no lateral shadows making the shadow underneath easily distinguished. Sunny scenes are lit by both sunlight and diffuse light casting lateral shadows. The shadow under the vehicle is noticeably darker than the lateral one because the latter is illuminated only by diffuse light. On a cloudy/rainy day, the street lighting could easily cause reflections from wet objects and asphalt, but the road under the vehicle is not affected, remaining dark and without brightness. In a tunnel the vehicle underneath is even darker than in other situations because artificial lighting is more direct and there is a low level of diffuse light, making the shadow practically black. 
The method most used to identify the shadow underneath the vehicle was proposed in [26]. A road area is extracted by defining the lowest central homogeneous region in the image "free driving space" delimited by edges. Then, a shadowed region is defined as a region that has lower intensity than a threshold value $m-3 \sigma$, where $m$ and $\sigma$ are the mean and variance of the road pixels' frequency distribution. This method has two important drawbacks. Firstly, the illumination conditions make the road's intensity vary non-uniformly. Even a well asphalted road can show zones where the pixels' intensity is significantly different. Secondly, not always the lowest central homogeneous region in the image matches with the road. In urban traffic, pedestrian crossings and sign markings, lateral shadows and patches of different asphalt are constantly appearing on the road and their edges are detected. The region delimited by edges may not belong to the road which can significantly mislead the vehicle detection procedure.

In order to overcome these drawbacks, we propose a thresholding method based on the histogram of only the ROI. A distinctive feature of the ROI image (Fig. 2a) is that its gray level histogram displays two characteristic peaks. The lower peak (nearest to 0 ) corresponds to the shadow underneath the vehicle and the higher one to the road. Intensity values due to lateral shadows, potholes, manhole covers, etc. can occur between the two peaks. As road markings are brighter (white, yellow) than the road, their intensity attains high values located on the histogram's right. Depending on the lighting, both peaks undergo gray level variation but the peak corresponding to the shadow does not attain values higher than 50 units in the histogram. The shadow's intensity values can vary between 0 (dark day) and 50 (clear day). This fact was throughout the system development and was confirmed in all tests. The pixel values obtained are specific to the sensor camera employed and depend on parameters such as pixel depth, dynamic range and exposure time. The thresholding criteria for the shadow's segmentation is to automatically choose the higher gray scale value (value on the right) of the lower intensity peak, as long as the latter is lower than 50 . In the cases where there is a vehicle in the ROI, the shadow's gray level peak is always present in the histogram and the shadow is easily segmented. 


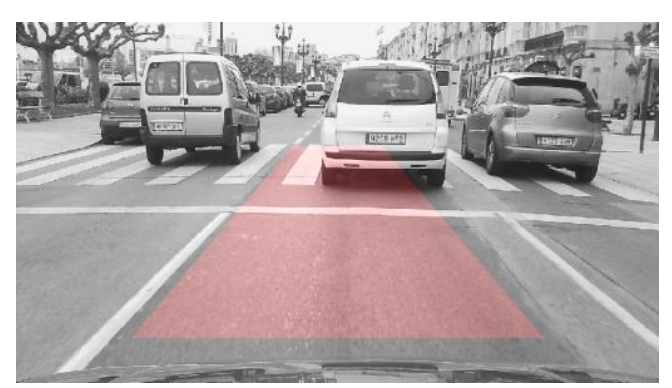

a

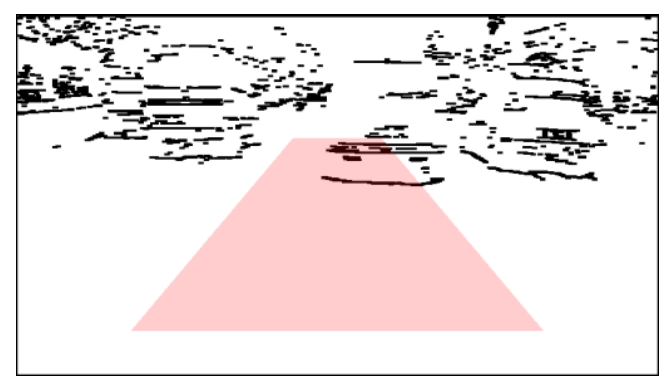

C

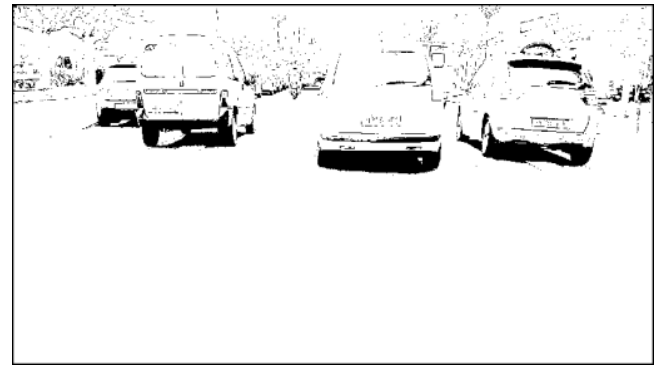

b

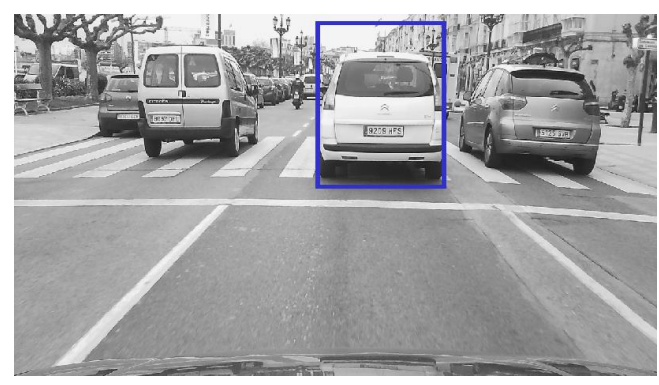

d

Fig. 2. Road scene. The region of interest ROI (a), the thresholded image (b), horizontal edges (c) and ROl's vehicle candidate (d).

In the absence of vehicles, the lower peak is not present and the intensity values are higher than 50 so the threshold is not established. In the case of a false shadow detection, the error is suppressed in the number plate detection stage, as the system does not detect any license plate. Fig. $2 \mathrm{~b}$ shows the shadowed regions of the whole scene whose values are below the threshold. After shadow thresholding, horizontal edges that correspond to the transitions from non-shadow region (bottom) to shadow regions (up) are extracted as in [26] and candidates are determined based on the location of those horizontal edges within the ROI (Fig. 2c). Only horizontal edges detected within the ROI, either in whole or in part, are considered while all those outside the ROI are discarded. 
Next, the bounding box containing the vehicles' back is obtained. As the dimensions of the vehicles' back are different for each make and model, a standard aspect ratio of vehicles' backs is assumed as in [26]. In this approach we consider that the length of the shadow's horizontal edge detected is the vehicle's width, and in order to encompass all kinds of vehicles and vans, the height of the box is equal to $130 \%$ of its width (Fig. 2 d).

Finally, as the shadow is on the road plane and assuming flat earth as in [17], a first rough estimation of the vehicle's distance is obtained based on the location of the lower edge of the vehicle's bounding box (the shadow's lower edge) in the image. This approximate distance is very useful because it in turn provides values of the vehicle number plate dimensions at this distance which are exploited in the number plate detection algorithm (section 3.5). The procedure is based on the relationship between the vertical location of the shadow in the image (in pixels) and the real vehicle's distance (meters). This relationship was established before the system was put into use and it also relates the vehicle's distance with the dimensions of the vehicle number plate characters (in pixels). This relationship is specific to the image resolution adopted, to the camera elevation in the ego-vehicle and to the camera tilt. To carry out this operation our vehicles were placed behind one another at a known distance (Dist), an image was taken and the shadow's vertical location (SVP) and the number plate's dimensions in the image were checked (Table 1). This process was done for different distances in a range from 1 to $10 \mathrm{~m}$ on different days to take into account different lighting conditions.

As can be observed in Table 1, for a similar vehicle distance (Dist), the vertical position of the shadow in the image (SVP) varies depending on the lighting conditions. This is basically due to two factors: firstly, the shadow underneath does not perfectly match with the vehicle's vertical projection onto the road. This factor is emphasized depending on the perspective which varies with the distance (the point of view is at a higher angle as the vehicle ahead becomes closer to the camera). Secondly, in sunny scenes there is a shadow around the vehicle (lateral shadow). There is not a clear intensity limit between the shadow underneath the vehicle and the 
surrounding shadow because the intensity values between both vary smoothly. In these cases it is very difficult to establish an automatic threshold which perfectly separates both shadows and inevitably some pixels belonging to the surrounding shadow are included as part of the shadow underneath. Taking these factors into account, the distance provided by the vehicle's shadow is not accurate enough, particularly for a close range and in sunny scenes. However, this approximate distance provides indicative values of the vehicle's number plate dimensions at this distance, making the next number plate detection method adaptive to the range (section 3.5).

Table 1 Vehicle's distance-Shadow's vertical location-Number plate character's dimensions

\begin{tabular}{|c|c|c|c|c|c|c|c|c|}
\hline \multirow{2}{*}{ Dist (m) } & \multicolumn{3}{|c|}{ SVP (pixel) } & \multirow{2}{*}{$\begin{array}{l}\text { NPW } \\
\text { (pixel) }\end{array}$} & \multirow{2}{*}{$\begin{array}{c}\mathrm{CH} \\
\text { (pixel) }\end{array}$} & \multirow{2}{*}{$N P W / C H$} & \multirow{2}{*}{$\begin{array}{c}\text { CS } \\
\text { (pixel) }\end{array}$} & \multirow{2}{*}{$\begin{array}{c}\mathrm{CT} \\
\text { (pixel) }\end{array}$} \\
\hline & Sunny & Cloudy & Rainy & & & & & \\
\hline 1 & 65 & 86 & 92 & 188 & 38 & 4.94 & 29 & 5 \\
\hline 2 & 119 & 137 & 142 & 138 & 27 & 5.11 & 21 & 4 \\
\hline 3 & 216 & 231 & 235 & 109 & 22 & 4.95 & 17 & 4 \\
\hline 4 & 282 & 294 & 297 & 91 & 19 & 4.78 & 15 & 3 \\
\hline 5 & 332 & 341 & 343 & 78 & 16 & 4.87 & 13 & 3 \\
\hline 6 & 371 & 378 & 380 & 69 & 14 & 4.92 & 11 & 2 \\
\hline 7 & 400 & 404 & 405 & 61 & 12 & 5.1 & 10 & 2 \\
\hline 8 & 426 & 428 & 428 & 55 & 11 & 5 & 9 & 2 \\
\hline 9 & 445 & 445 & 445 & 50 & 10 & 5 & 8 & 1 \\
\hline 10 & 465 & 465 & 465 & 45 & 9 & 5 & 7 & 1 \\
\hline
\end{tabular}

Dist $=$ Real vehicle's distance, SVP = Vertical position of the lower edge of the vehicle's bounding box (shadow) in the image, NPW =Number plate joint width, $\mathrm{CH}=$ Character height, $\mathrm{CS}=$ Separation between numbers and letters, CT= Character thickness trace.

\subsection{Number Plate Features and Distance-Size Relationship}

The aim of the next license plate detection is to calculate vehicle distance to the camera and therefore the vehicle's distance. License plates have several constant parameters that can be checked in order to obtain the distance. The longer the dimensions, the more accurate the measurement. The ideal dimension to be checked would be the plate's width. Nevertheless, experience indicated that with light colored vehicles the result of the image processing is not satisfactory when the aim is to obtain the plate's contour. However, the plate's characters can be easily localized and isolated by means of morphological methods. The system proposed was 


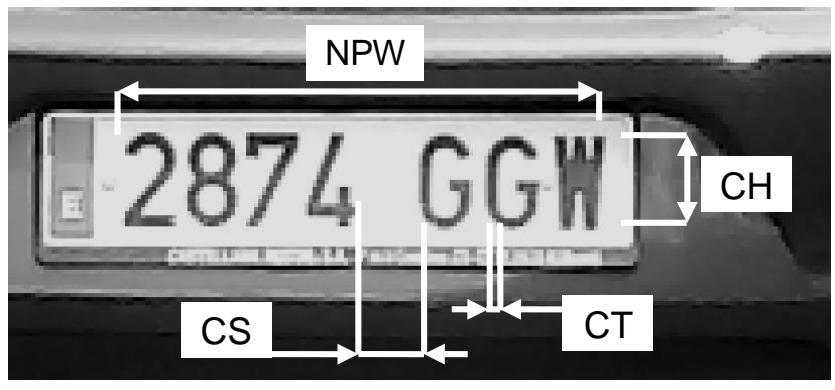

Fig. 3. Number plate parameters. NPW = Number plate width, $\mathrm{CH}=$ Character height, $\mathrm{CT}=$ Character thickness trace, $\mathrm{CS}=$ Characters separation.

designed to work with Spanish plates but it can be adapted to plates of other countries. Spanish plates are made up of a four numbers and three letters, and their dimensions are fixed (Fig. 3). In order to calculate the vehicle's distance, two dimensions of its number plate are considered by the algorithm: the width of the number plate (NPW) and the height of the characters $(\mathrm{CH})$. The consideration of one or the other depends on the skew angle of the vehicle ahead. When the back of the vehicle ahead is in the frontal view, the parameter considered to estimate the distance is the NPW. In this case both parameters could be employed in the measurement but as the NPW is longer than the $\mathrm{CH}$, the accuracy provided in the distance measurement is greater.

However, when the vehicle ahead is on a curve, the image is not a perfect frontal view of the vehicle's rear so the NPW in the image is shorter than it should be, which generates a distance measuring error. In these cases, the number plate parameter considered to establish the vehicle's distance is the height $(\mathrm{CH})$ of the nearest character (the highest). In a skewed situation the characters of the plate do not have the same size. If a rotation of the plate were performed in order to place the plate in a frontal view, the axis of this rotation would be the highest side of the highest character of the skewed plate. Furthermore, after this rotation the height of the number plate in a frontal view would be the same as the height of the highest character of the skewed number plate, so this rotation is unnecessary. 
In order to know if the rear of the vehicle ahead is in a frontal view, the algorithm makes use of the aspect constant relationship between the two parameters in a frontal view:

$$
\frac{N P W}{C H} \approx 5=c t e
$$

The relationships between the NPW, $\mathrm{CH}$, aspect constant and the distance to the camera were established in Table 1. Fig. 4 is the graphical representation of NPW, CH and the distance.

From Fig. 4, two mathematical relationships were obtained:

$$
\begin{gathered}
D_{N P W}=24.8 e^{(-0.03954 N P W)}+10.18 e^{(-0.01231 N P W)} \\
D_{C H}=42.59 e^{(-0.3508 C H)}+16.32 e^{(-0.07613 C H)}
\end{gathered}
$$

where $D_{N P W}(m)$ is the vehicle distance provided by the width of the number plate and $D_{C H}(m)$ is the vehicle distance provided by the character's height. Fig. 4 shows how NPW and CH do not vary linearly, but decrease exponentially with the distance.

Table 1 shows how the aspect relationship of the NPW and the $\mathrm{CH}$ in frontal view remains practically constant and equal to 5 . Moreover, Table 1 shows the different accuracy provided by NPW and $\mathrm{CH}$. For instance, from 5 to $6 \mathrm{~m}$ the use of the NPW provides a measurement precision of $0.11 \mathrm{~m}(1 \mathrm{~m} / 9 \mathrm{pix})$, while the $\mathrm{CH}$ provides a precision of $0.33 \mathrm{~m}(1 \mathrm{~m} / 3 \mathrm{pix})$.

\subsection{Number Plate Detection}

The number plate detection procedure proposed is based on the well-known morphological operator, Top-Hat. This method is widely employed in number plate localization under restricted conditions where some information related to the number plate's dimensions in the image is available. We make this method adaptive to vehicles in motion at any distance within the range. The number plate detection is restricted to the vehicle's bounding box, thereby significantly simplifying the background region. The Top-Hat operator is described as:

$$
D(i, j)=|I(i, j)-C(i, j)|
$$




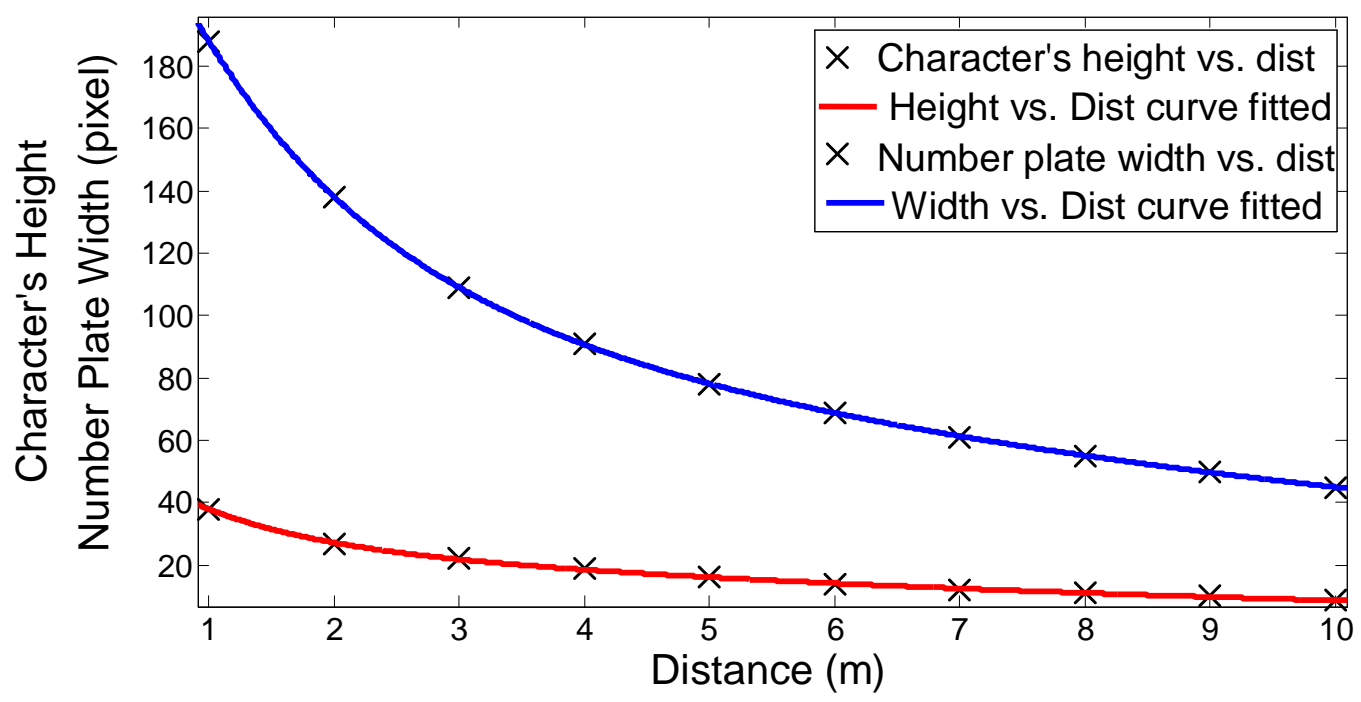

Fig. 4. Character's height vs. distance and its fitting curve. Number plate width vs. distance and its fitting curve.

Firstly, the morphological closing $C$ of the image $I$ with a circular structuring element (SE) eliminates all the dark on light background elements smaller than SE (Fig. 5b). Then, subtracting

the resulting image from the initial one, we get an image $D$ where non filtering sensitive elements are removed and the high frequency areas (including the plate's characters) remain enhanced (see Fig. 5c).

The size of the structuring element used is crucial and should be estimated according to the plate's font size (i.e. to the characters' thickness) in the image. Since the number plate characters' thickness in the image varies significantly depending on the distance to the camera, a constant SE cannot be considered as in [35-37]. In order to make this method adaptive to the range, we take advantage of the lower edge location of the vehicle's bounding box (section 3.3). Table 1 establishes a relationship between this lower edge location in the image (SVP), the real vehicle's distance (Dist) and the character's thickness (CT). In Table 1, the structuring element SE 
considered by the algorithm is the value of the CT corresponding to the minimum Dist nearest to the SVP value provided by the vehicle detection procedure. In this way, the SE is slightly higher than the character's trace at the real vehicle distance what ensures an adequate performance of the morphological method.

After the operator Top-Hat is applied, the binarization threshold of the still gray image is established by means of the Otsu method [38] as in [32, 35]. Fig. $5 d$ is the binary image obtained, and, apart from the number plate, other noisy elements whose trace is smaller than the SE diameter inevitably appear. In order to eliminate them and isolate the number plate characters, the algorithm is based on a morphologic filter, making use of the knowledge regarding the number plate dimensions expected at each approximate distance. Table 1 also establishes the relationship between the parameters previously mentioned and the character's height $(\mathrm{CH})$, the number plate width (NPW) and the separation between the number and letters (CS). As stated above, the parameters' values provided by the SVP are slightly higher than the parameters' values at the real vehicle's distance, which ensures a correct performance of the morphological filter.

Firstly, all the elements are horizontally joined by means of a 'closing' operation with a horizontal linear SE of value CS which is the maximum separation between the plate's characters (see Fig. 3). Thus, all elements whose horizontal separation is less than or equal to the SE are joined horizontally (Fig. 6a). Next, object size discrimination is performed as in [35]. Elements taller than $\mathrm{CH}$ and wider than NPW are eliminated by means of an 'opening' operation followed by the subtraction of the resulting 'opening' image from the former one. This operation is applied two times, firstly with a vertical linear SE of value $\mathrm{CH}$ and secondly with a horizontal linear SE of value NPW (Fig. 6b). Note that the two size restrictions are always satisfied by both frontal view and skewed number plates as the latter's parameters are always smaller ( $\mathrm{CH}$ can be equal) than the former's ones. Next, small element discrimination is performed by means of an 'opening' operation. There is not reference values slightly lower neither than $\mathrm{CH}$ nor NPW so a safety 


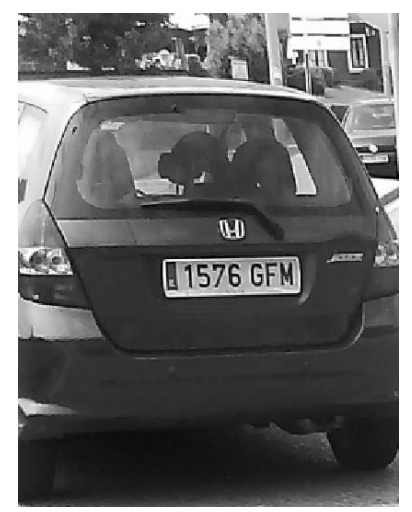

a

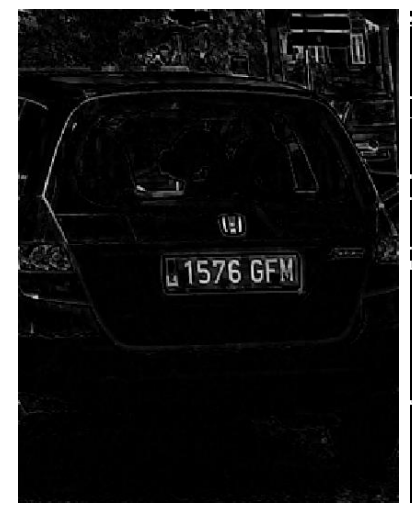

C

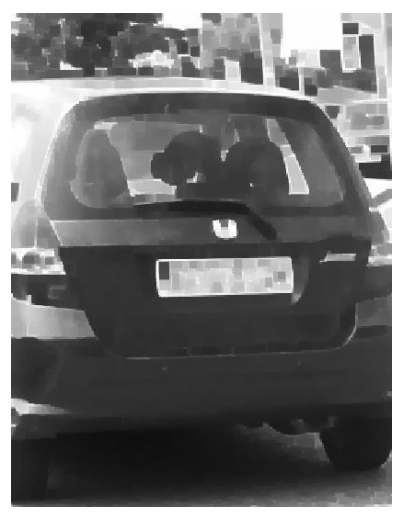

b

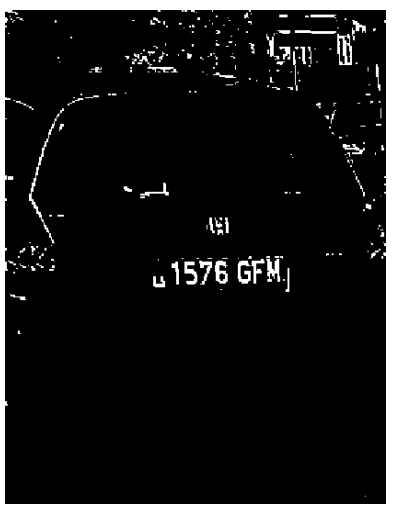

d

Fig. 5. Vehicle candidate (a), morphological closing with SE (b), resulting image of the top hat method $(c)$, the resulting binary image (d).

percentage is considered as the lower limit for both dimensions. This operation is applied two times, firstly with a vertical linear SE of value $0.7 \mathrm{CH}$ and secondly with a horizontal linear SE of

value 0.6NPW (see Fig. 6c). As can be observed in Fig.6c, number plate candidates are contained in rectangular regions which satisfy the conditions of height, width and maximum separation among elements.

Finally, the number plate hypothesis verification is based on three new restrictions. Firstly, number plates are made up of seven characters (four numbers and three letters). Secondly, the height of all of them is the same (in skewed number plates the characters' height is not equal but approximate) and thirdly, the separation between the numbers and the letters (CS) is greater than 
the separation between individual numbers and letters. In order to evaluate these restrictions an 'AND' operation is performed between the candidate regions and their corresponding coordinates of the initial binary image. The result is the elements contained in the rectangular candidates (Fig. $6 d)$. A four-connected-component algorithm is used in each region obtaining as a result all the elements labeled. Finally, after obtaining the number of elements, their separation and their height, the candidate which satisfies the restrictions imposed is verified as the number plate.

\subsection{Distance Measurement}

The penultimate stage of the system consists of extracting the width of the seven characters of the number plate (real NPW) and the tallest character height (real $\mathrm{CH}$ ). The aspect ratio between the two parameters is obtained from equation (1) and the result is compared with the aspect ratio parameter given by the SVP in Table 1 . If the difference between them is les than $5 \%$, the scene is considered a perfect frontal view so the vehicle's distance is obtained in a straightforward manner from equation (2) by means of the real NPW. In any other case, the scene is skewed so the vehicle's distance is directly obtained from equation (3) by means of the real $\mathrm{CH}$.

\subsection{Relative Speed Measurement}

In order to calculate the front vehicle's relative speed, the system considers successive images. Knowing the vehicle distance variation in two consecutive images and the time elapsed between each image acquisition, the vehicle's relative speed is calculated as:

$$
V_{\text {relative }}=\frac{D_{2} \quad D_{1}}{\Delta T}
$$

where D1 is the vehicle distance in the first frame, D2 is the vehicle distance in the second frame and $\Delta T$ is the time between the two frames. 


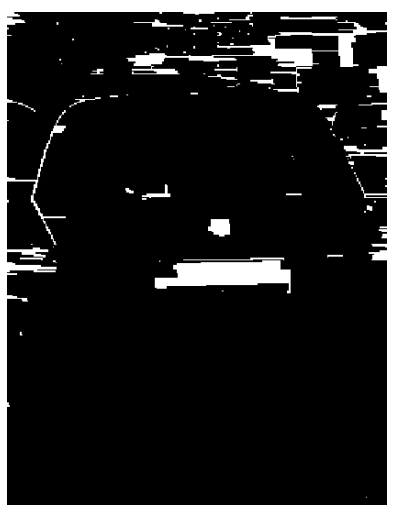

a

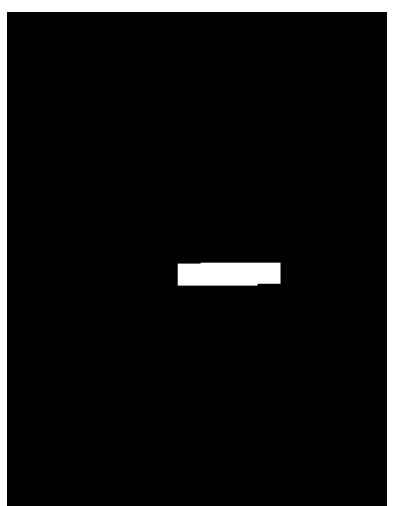

C

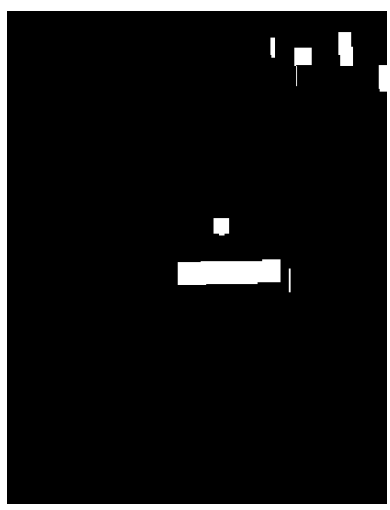

b

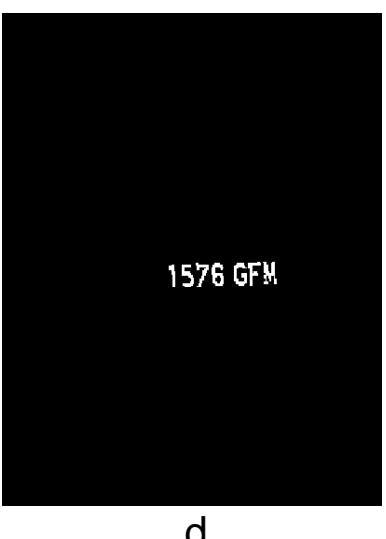

d

Fig. 6. Elements horizontally joined (a), image without bigger noisy elements (b), image without smaller noisy elements (number plate candidates) (c), elements contained in the rectangular candidate (d).

\section{EXPERIMENTAL RESULTS}

The system was installed on a vehicle and it was subjected to a set of tests under different traffic and lighting situations in Santander, Spain. The camera used was a Trust Widescreen HD WebCam, 8 bit pixel depth, auto white balance and automatic exposure time which provided grayscale $1280 \times 720$ frames. The images were acquired and processed in Matlab ${ }^{\circledR}$, on an Intel Pentium IV $3.06 \mathrm{GHz}$ with 480 MB RAM. 


\subsection{Distance Accuracy}

In order to check the distance measurement accuracy, a comparison between the measurements provided by the vision system and a laser was performed. The laser was a Leica Disto Pro A distance meter with a range up to $100 \mathrm{~m}$. Tests consisted of placing our vehicle behind a target vehicle in a range from 0.5 to $10 \mathrm{~m}$ at intervals of $0.5 \mathrm{~m}$. Both vehicles were at rest and the measurements were taken by both the monocular system and the laser. The measurement provided by the laser was considered the real distance. At each point, the mean of 10 measures was calculated. The algorithm correctly estimated that the scene was a frontal view so the distance was provided by the width of the number plate (NPW Dist) over the whole range. In order to check the accuracy that the character's height ( $\mathrm{CH}$ Dist) would provide in a curve, the

Table 2 Monocular Vision System-Laser

\begin{tabular}{ccccccc}
\hline \multirow{2}{*}{ Laser Dist } & \multicolumn{2}{c}{ Cloudy } & \multicolumn{2}{c}{ Sunny } & \multicolumn{2}{c}{ Rainy } \\
\cline { 2 - 7 } & NPW Dist & CH Dist & NPW Dist & CH Dist & NPW Dist & CH Dist \\
\hline 0.5 & 0.500 & 0.51 & 0.494 & 0.51 & 0.506 & 0.51 \\
1 & 1.020 & 0.97 & 1.033 & 0.97 & 1.007 & 0.97 \\
1.5 & 1.484 & 1.46 & 1.503 & 1.46 & 1.464 & 1.46 \\
2 & 1.967 & 2.09 & 1.995 & 2.09 & 1.941 & 2.09 \\
2.5 & 2.466 & 2.63 & 2.502 & 2.63 & 2.431 & 2.63 \\
3 & 2.994 & 3.07 & 3.040 & 3.07 & 2.948 & 3.07 \\
3.5 & 3.504 & 3.32 & 3.561 & 3.32 & 3.448 & 3.32 \\
4 & 3.999 & 3.89 & 3.999 & 3.89 & 3.932 & 3.89 \\
4.5 & 4.515 & 4.58 & 4.515 & 4.58 & 4.436 & 4.58 \\
5 & 5.032 & 4.98 & 5.032 & 4.98 & 4.940 & 4.98 \\
5.5 & 5.527 & 5.43 & 5.527 & 5.43 & 5.423 & 5.43 \\
6 & 5.974 & 5.93 & 5.974 & 5.93 & 5.857 & 5.93 \\
6.5 & 6.471 & 6.51 & 6.471 & 6.51 & 6.471 & 6.51 \\
7 & 7.027 & 7.17 & 7.027 & 7.17 & 7.027 & 7.17 \\
7.5 & 7.488 & 7.17 & 7.488 & 7.17 & 7.488 & 7.17 \\
8 & 7.990 & 7.96 & 7.990 & 7.96 & 7.990 & 7.96 \\
8.5 & 8.540 & 7.96 & 8.540 & 7.96 & 8.540 & 7.96 \\
9 & 8.935 & 8.89 & 8.935 & 8.89 & 8.935 & 8.89 \\
9.5 & 9.574 & 8.89 & 9.574 & 8.89 & 9.574 & 8.89 \\
10 & 10.035 & 10.03 & 10.035 & 10.03 & 10.035 & 10.03 \\
\hline
\end{tabular}

Laser Dist= Distance provided by the Laser, NPW Dist= Distance provided by the width of the number plate, $\mathrm{CH}$ Dist= Distance provided by the character's height. 
character's height in the recorded images was analyzed in the laboratory. The results for tests in sunny, cloudy and rainy days were in agreement (Table 2).

Table 2 shows that the Laser Dist and the NPW Dist remained practically the same regardless of lighting, the results varying slightly closer than $5.5 \mathrm{~m}$. However, as expected, the accuracy provided by the character's height was lower. The absolute and relative errors were calculated for each distance in sunny, cloudy and rainy conditions from Table 2. As an example, Fig. 7 shows the graphical representation of the measurements provided by the laser and the monovision system in a cloudy day. Fig. 7a shows that the measurement difference (absolute error) between the real and the NPW Dist is small for the whole range, providing very accurate measurements. In Fig. $7 \mathrm{~b}$ the absolute error of the $\mathrm{CH}$ Dist is greater the longer the range and it can be appreciated that the relative error gets higher as the distance becomes longer.

Table 3 shows the absolute and relative errors of the NPW and CH distances in cloudy conditions. As can be observed, the maximum relative error of the NPW Dist is $2.00 \%$ at $1 \mathrm{~m}$ where the absolute error is only $0.020 \mathrm{~m}$. For $10 \mathrm{~m}$ the absolute error is $0.035 \mathrm{~m}$ which implies $0.35 \%$ relative error. Regarding $\mathrm{CH}$ Dist, Table 3 shows that from $0.5 \mathrm{~m}$ to $7 \mathrm{~m} \mathrm{CH}$ provides an absolute error lower than $0.02 \mathrm{~m}$, however from $7 \mathrm{~m}$ to $10 \mathrm{~m}$ the $\mathrm{CH}$ distance accuracy decreases significantly in comparison to NPW distance. The accuracy would improve for both parameters with a higher image resolution although processing time would be longer. Tests showed that as expected, the accuracy provided by NPW Dist is higher than the CH Dist's and they also showed that the algorithm performs correctly regardless of lighting conditions and estimates correctly whether the scene is a frontal view.

\subsection{Vehicle and Number Plate Detection}

The second test was the evaluation of vehicle and number plate detection processes. Tests were carried out in real urban traffic on cloudy, sunny, rainy days and in a tunnel. Due to the incorrect 


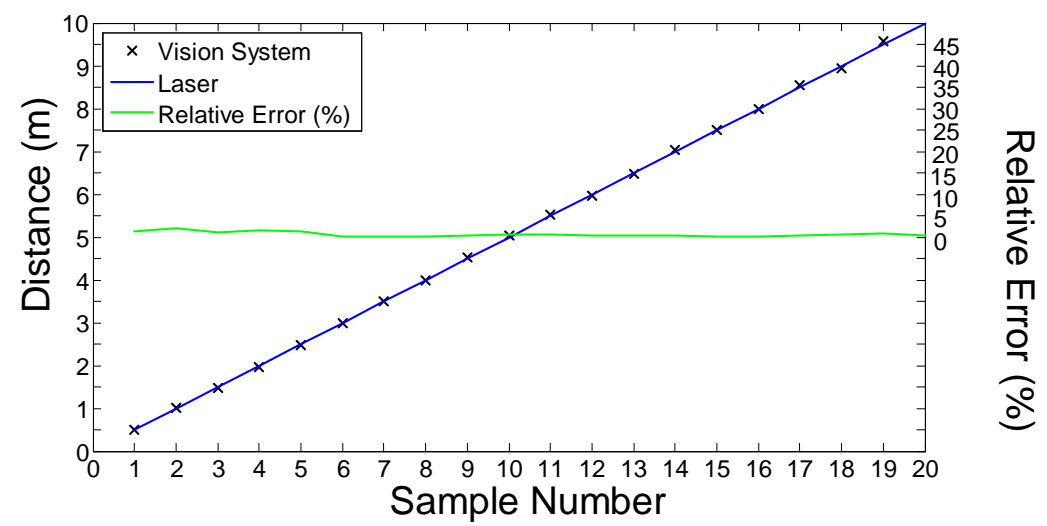

a

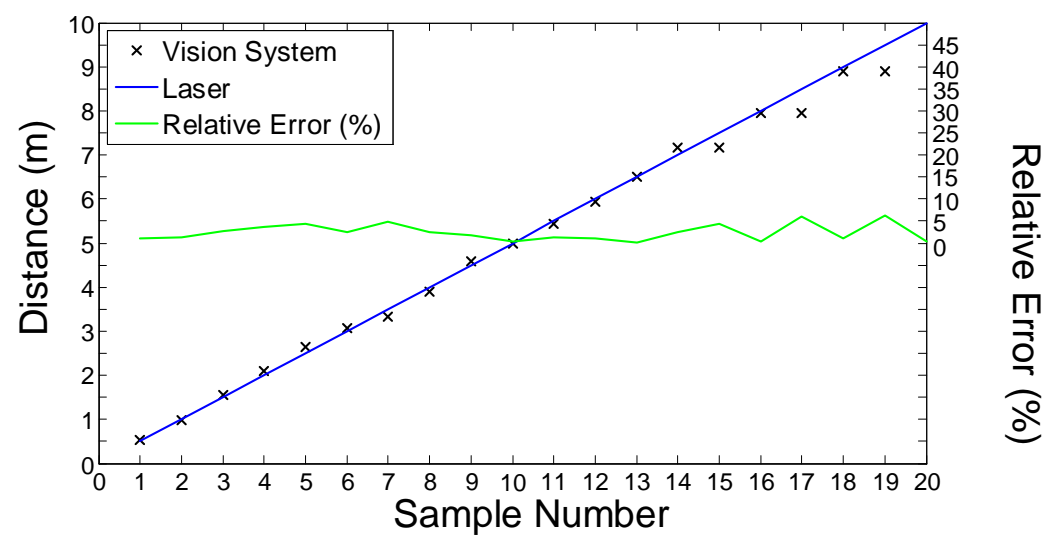

b

. 7. Distance measures by monocular system - laser and relative error. NPW Vs. Laser (a). CH Vs. Laser (b).

performance in motion of the laser distance meter, the distance measurement comparison could not be carried out. 1000 images in 5 sets of 200 images were acquired and recorded for each

weather situation and 400 in 4 sets of 100 in a tunnel. The results of the vehicle and number plate detection are presented in Table 4-5 respectively.

Vehicle detection results showed the effectiveness and excellent performance of the algorithm proposed, with a high percentage in the Vehicle Detection Rates (VDR) achieving 98.35\%, $99.20 \%, 98.64 \%$ and $97.38 \%$ for cloudy, sunny, rainy days and tunnel respectively. Moreover, False Detection Rates (FVDR) were very low making the system very reliable. 
Regarding license plate detection (Table 5), the detection rates (LPLR) were over 99\%. It was very high in tunnels $(99.40 \%)$ because the plate's background is made of reflective material, providing high contrast with the characters when it is lit by headlights. Moreover, there was no any

Table 3 Analysis of Errors under Cloudy Conditions

\begin{tabular}{ccccc}
\hline Laser Dist $(m)$ & NPW Absolute Error $(m)$ & NPW Relative Error (\%) & CH Absolute Error $(m)$ & CH Relative Error (\%) \\
\hline 0.5 & 0.000 & 0.00 & 0.011 & 2.22 \\
1 & 0.020 & 2.00 & 0.024 & 2.40 \\
1.5 & 0.016 & 1.06 & 0.040 & 2.66 \\
2 & 0.032 & 1.60 & 0.092 & 4.63 \\
2.5 & 0.033 & 1.33 & 0.134 & 2.39 \\
3 & 0.006 & 0.19 & 0.076 & 4.97 \\
3.5 & 0.004 & 0.11 & 0.173 & 2.59 \\
4 & 0.000 & 0.00 & 0.104 & 1.84 \\
4.5 & 0.015 & 0.33 & 0.083 & 1.27 \\
5 & 0.032 & 0.64 & 0.017 & 1.08 \\
5.5 & 0.027 & 0.50 & 0.069 & 0.17 \\
6 & 0.43 & 0.065 & 2.54 \\
6.5 & 0.026 & 0.44 & 0.011 & 4.28 \\
7 & 0.028 & 0.39 & 0.178 & 0.47 \\
8 & 0.027 & 0.15 & 0.321 & 6.32 \\
8.5 & 0.011 & 0.11 & 0.038 & 1.13 \\
9 & 0.009 & 0.47 & 0.538 & 6.33 \\
10 & 0.040 & 0.71 & 0.101 & 0.37 \\
\hline
\end{tabular}

AbsoluteError $(\mathrm{m})=$ LaserDist- Dist; RelativeError $(\%)=\frac{\text { LaserDist Dist }}{\text { LaserDist }} \cdot 100$

False License Plate Localization (NFLPL), which makes the algorithm very robust. The excellent reliability of the license plate localization is because the algorithm only focuses on the bounding box image which only contains the rear of the vehicle ahead.

Finally, it must be said that the processing time of the system was not constant. It depends on the distance from the vehicle to the camera and the number of plate candidates. The closer to the camera, the bigger vehicle's rear image size, and so the longer the processing time. The average processing time achieved was $248.4768 \mathrm{~ms}$, which implies $4 \mathrm{fps}$. It is due to the limitations imposed by the processing unit employed, a common laptop. However, it must be said that the image processing algorithm developed, employs methods and techniques that are much faster than processes such as image corresponding of stereovision, which is usually employed in 
approaches that satisfy the timing condition. The algorithm is expected to be able to achieve a higher number of fps running on a suitable processing unit [39].

\section{CONCLUSION}

A novel monovision-based system able to detect a vehicle ahead and measure the distance and relative speed has been presented. The use of a single common camera makes the system cheaper than stereovision systems and other technologies such as RADAR-based approaches.

Table $5 \quad$ Analysis of License Plate Detection

\begin{tabular}{cccccccc}
\hline & $\begin{array}{c}\text { Total } \\
\text { Number of } \\
\text { Test Frames }\end{array}$ & $\begin{array}{c}\text { Number of Frames } \\
\text { with Vehicle in the } \\
\text { Safety Area (NFV) }\end{array}$ & $\begin{array}{c}\text { Number of } \\
\text { Vehicles } \\
\text { Detected } \\
(N V D)\end{array}$ & $\begin{array}{c}\text { Number of LP } \\
\text { Localizations } \\
(N L P L)\end{array}$ & $\begin{array}{c}\text { Number of False } \\
\text { LP Localizations } \\
(N F L P L)\end{array}$ & $\begin{array}{c}\text { License } \\
\text { Plate } \\
\text { Localization } \\
\text { Rate }(L P L R)\end{array}$ & $\begin{array}{c}\text { False License } \\
\text { Plate } \\
\text { Localization } \\
\text { Rate }(F L P L R)\end{array}$ \\
\hline Cloudy & 1000 & 486 & 478 & 475 & 0 & $99.37 \%$ \\
Sunny & 1000 & 502 & 498 & 494 & 0 & $0 \%$ \\
Rainy & 1000 & 515 & 508 & 504 & 0 & $99.19 \%$ \\
Tunnel & 400 & 344 & 335 & 334 & 0 & $99.21 \%$ & $0 \%$ \\
\hline
\end{tabular}

$\mathrm{NFV}=$ Number of Frames with Vehicle in the Safety Area, NVD=Number of Vehicles Detected, NLPL=Number of License Plate Localizations, NFLPL $=$ Number of False License Plate Localizations, LPLR= License Plate Localization Rate, FLPLR $=$ False License Plate Localization Rate.

$\operatorname{LPLR}(\%)=\frac{\mathrm{NLPL}}{\mathrm{NVD}} \quad 100 \quad$ FLPLR $(\%)=\frac{\text { NFLPL }}{\text { NFD }} \quad 100$

Table 4 Analysis of Vehicle Detection

\begin{tabular}{|c|c|c|c|c|c|c|c|}
\hline & $\begin{array}{c}\text { Total } \\
\text { Number of } \\
\text { Test Frames }\end{array}$ & $\begin{array}{l}\text { Number of Frames } \\
\text { with Vehicle in the } \\
\text { Safety Area (NFV) }\end{array}$ & $\begin{array}{c}\text { Number of Frames } \\
\text { with no Vehicle in } \\
\text { the Safety Area } \\
\text { (NFNV) }\end{array}$ & $\begin{array}{c}\text { Number of } \\
\text { Vehicles } \\
\text { Detected } \\
(N V D) \\
\end{array}$ & $\begin{array}{l}\text { Number of } \\
\text { False } \\
\text { Detections } \\
(N F D) \\
\end{array}$ & $\begin{array}{c}\text { Vehicle } \\
\text { Detection } \\
\text { Rate }(V D R)\end{array}$ & $\begin{array}{c}\text { False Vehicle } \\
\text { Detection Rate } \\
\text { (FVDR) }\end{array}$ \\
\hline Sunny & 1000 & 502 & 498 & 498 & 4 & $99.20 \%$ & $0.80 \%$ \\
\hline Rainy & 1000 & 515 & 485 & 508 & 7 & $98.64 \%$ & $1.44 \%$ \\
\hline Tunnel & 400 & 344 & 56 & 335 & 2 & $97.38 \%$ & $3.57 \%$ \\
\hline
\end{tabular}

VDR=Vehicle Detection Rate, FVDR=False Vehicle Detection Rate, NFV=Number of Frames with Vehicle in the Safety Area, $N F N V=N u m b e r$ of Frames with No Vehicle in the Safety Area, NVD=Number of Vehicles Detected, NFD=Number of False Detections.

$\operatorname{VDR}(\%)=\frac{\text { NVD }}{\text { NFV }} \quad 100 \quad$ FVDR $(\%)=\frac{\text { NFD }}{\text { NFNV }} \quad 100$

Besides, monocular vision significantly reduces the computational complexity and the processing time of stereovision. The distance measurement method proposed is based on the vehicle's number plate whose dimensions and shape are standardized in each country. The algorithm simplifies the complex traffic scene focusing only on a ROI of the road corresponding to the safety area in front of our vehicle. The ROI reduces the possibility of errors improving the system's 
reliability. The vehicle detection procedure successfully utilizes the shadow underneath the target vehicle and horizontal edges regardless of weather conditions. An adaptive shadow segmentation threshold is proposed based on the characteristic ROI image histogram. The number plate localization algorithm proposed adapts the Top-Hat operator to vehicles in motion over the range. In-vehicle tests carried out in real urban traffic showed excellent robustness and reliability in vehicle and number plate detection and very good accuracy in distance measurement.

\section{ACKNOWLEDGMENT}

This work is funded by Spanish Ministry of Innovation and Science. (Project: DPI2012-36959).

\section{REFERENCES}

[1] E. Bertolazzi, F. Biral, M. Da Lio, A. Saroldi, F. Tango: 'Supporting Drivers in Keeping Safe Speed and Safe Distance', IEEE Trans. Intell. Trans. Systems, Sept. 2010, vol. 11, no. 3, pp. 525-538.

[2] G. Toulminet, M. Bertozzi, S. Mousset, A. Bemsrhair, A. Broggi: 'Vehicle Detection by Means of Stereo Vision-Based Obstacles Features Extraction and Monocular Pattern Analysis', IEEE Trans. Intell. Trans. Systems, August. 2006, vol. 15, no. 8, pp. 2364-2375.

[3] M. Skutek, M. Mekhaiel, G. Wanielik: 'A PreCrash System based on Radar for Automotive Applications', Proc. IEEE of Intelligent Vehicles Symposium, Columbus, 2003, pp.37-41.

[4] S. Velupillai, L. Guvenc: 'Laser Scanners for Driver-Assistance Systems in Intelligent Vehicles', IEEE Control Systems Magazine, 2009, vol. 29, no. 2, pp.17-19.

[5] L. Alonso, J.P. Oria, M. Fernández, C. Rodríguez, J. Arce, M. Ibarra, V. Ordoñez: ‘Genetically Tuned Controller of an Adaptative Cruise Control for Urban Traffic Based on Ultrasounds', Lecture Notes in Computer Science, 2010, vol. 6553, pp. 479-485.

[6] S. Nedevschi, R. Danescu, et al: 'High accuracy stereovision approach for obstacle detection on non planar roads', Proc. of IEEE Intelligent Engineering Systems (INES), Cluj Napoca, Romania, 2004, pp. 211-216.

[7] A. Broggi, M. Bertozzi, A. Fascioli, C. Guarino, A. Piazzi: 'Visual Perception of Obstacles and Vehicles for Platooning', IEEE Trans. on Intelligent Transportation Systems, 2000, vol. 1, no. 3, pp.164-176. 
[8] Z. Sun, G. Bebis, R. Miller: "On-road vehicle detection: a review", IEEE Transactions on Pattern Analysis and Machine Intelligence, 2006, vol. 28, n.5, pp. 694-711.

[9] A. Makris, M. Perrollaz, et al: 'Integration of visual and depth information for vehicle detection', Proc. of IEEE/RSJ International Conference on Intelligent Robots and Systems, San Francisco, USA, 2011.

[10] A. Amditis, E. Bertolazzi, M. Bimpas, et al: 'A Holistic Approach to the Integration of Safety Applications: The INSAFES Subproject Within the European Framework Programme 6 Integrating Project PReVENT', IEEE Trans. on Intelligent Transportation Systems, 2010, vol. 11, no. 3, pp. 554-566.

[11] T. Michalke, J. Fritsch, C. Goerick: 'A biologically-inspired vision architecture for resource-constrained intelligent vehicles', Computer Vision and Image Understanding, 2010, vol. 114, no. 5, pp. 548-563.

[12] V. Petrovic, T. Cootes: 'Analysis of features for rigid structure vehicle type recognition', Proc. of British Machine Vision Conference, 2004, pp. 587-596B.

[13] M. Bertozzi, A. Broggi, A. Fascioli, S. Nichele: 'Stereo Vision-based Vehicle Detection', Proc. IEEE Intelligent Vehicles Symposium, 2000, pp. 39-44.

[14] B. Leibe, K. schindler, et al: 'Couple Object Detection and Tracking from Static Cameras and Moving Vehicles', IEEE Trans. on Pattern Analysis and Machine Intell., 2008, vol. 30, no. 10, pp. 1683-1698.

[15] J. Michels, A. Saxena, A.Y.Ng: 'High Speed Obstacle Avoidance using Monocular Vision and Reinforcement Learning', 22nd Int. Conf. on Machine Learning, Bonn, Germany, 2005, pp. 593-600.

[16] I. Gat, M. Benady, A. Shashua: 'A Monocular Vision Advance Warning System for the Automotive Aftermarket', SAE Technical Paper, 2005.

[17] M. Nieto, J. Arróspide, D. Stavens, L. Salgado: 'Road environment modelling using robust perspective analysis and recursive Bayesian segmentation', Machine Vision and Appli., 2011 vol. 22, pp. 927-945.

[18] S. Álvarez, M. Á. Sotelo, M. Ocaña, D. F. Llorca, I. Parra, L. M. Bergasa: 'Perception advances in outdoor vehicle detection for automatic cruise control', Robotica, 2010, vol. 28, pp. 765-779.

[19] A. Kuehnle: 'Symmetry-based recognition for vehicle rears', Pattern Recognition Letters, 1991, vol 12, pp. 249-258.

[20] T. Kalinke, C. Tzomakas, W. von Seelen: 'A texture-based object detection and an adaptative model-based classification', Proc. of IEEE International Conference on Intelligent Vehicles, Sttutgard, 1998, pp143-148. 
[21] D. Guo, T. Fraichard, M. Xie, C. Laugier: 'Color modelling by spherical influence field in sensing driving environment', Proc. of IEEE Intelligent Vehicle Symposium, Dearborn, 2000, pp. 249-254.

[22] P. Paroli, G. Piccioli: 'A feature-based recognition scheme for traffic scenes', Proc. Intelligent Vehicle Symposium, Detroit, 1995, pp. 229-234.

[23] M. Bertozzi, A. Broggi, S. Castelluccio: 'A real-time oriented system for vehicle detection', Journal of Systems Architecture, 1997, pp. 317-325.

[24] T. Michalke, F. Stein, U. Franke: 'Towards a closer fusion of active and passive safety: Optical flow-based detection of vehicle side collisions', Proc. of Intel. Vehicles Symposium (IV), Stuttgart, 2011, pp. 181-188.

[25] H. Mori, N. Charkai: 'Shadow and Rhythm as Sign patterns of Obstacle Detection', Proc. of International Symposium on Industrial Electronics, Budapest, 1993, pp. 271-277.

[26] C. Tzomakas, W. Seelen: 'Vehicle Detection in Traffic Scenes using Shadows', Tech. Rep. 98-06, Institut FurNeuroinformatik, Ruht-universitat, Bochum, Germany, 1998.

[27] M.B. Van Leeuwen, F.C.A. Groen: 'Vehicle Detection with a Mobile Camera, Spotting Midrange, Distant and Passing Cars', IEEE Robotics and Automation Magazine, March, 2005, vol. 12, no.1, pp. 37-43.

[28] W. P. Yang, J. C. Li, Z. K. Shen: 'Technique of Automatic Orientation of Car License Plate Targets', Journal of Image and Graphics, 2002, vol. 7, pp.835-839.

[29] Y. Yanamura, M. Goto, et al: 'Extraction and Tracking of The License Plate Using Hough Transform and Voted Block Matching', Proc. of IEEE Intelligent Vehicles Symposium, 2003, pp.243-246.

[30] W. J. Li, D. Q. Liang, Q. Zhang, X. Fan: 'A Novel Approach for Vehicle License Plate Location Based on Edge-Color Pair', Chinese Journal of Computers, 2004, 27(2), pp. 204-208.

[31] G. Q. Liu, X. S. Zhang X. B. Zhang: 'License Plate Location Based on Texture Characteristic of Image', Journal of Image and Graphics, 2005, 10(11), pp.1420-1423.

[32] P. V. Suryanarayana, S. K. Mitra, A. Banerjee, A. K. Roy: 'A Morophology Based Approach for Car License Plate Extraction', IEEE Indicon Conference, Chennai, India, 2005, pp.24-27.

[33] K. K. Kim, K. I. Kim, J. B. Kim, H. J. Kim: 'Learning-based Approach for License Plate Recognition', IEEE Neural Network for Signal Processing Workshop 2000, Sydney, Australia, 2000, pp.614-623.

[34] H. Chengwen, Z. Yannan, W. Jiaxin, Y. Zehong: 'An improved method for the Character recognition based on SVM', Proc. of the IASTED Int. Conf. On Artificial Intell. And Applications, 2006, pp.457-461. 
[35] F. Martín, M. Garcia, J.L. Alba: 'New Methods for Automatic Reading of VLP's', Proc. of the IASTED Int. Conf. on Signal Processing, Pattern Recognition and Applications, SPPRA, 2002.

[36] W. Li, Z. Zhu, Z. Jiang: 'A New Method for License Plate Location Based on Top-Hat Transform and Wavelet Transform', Int. Conf. in Innovative Computing, Inform. and Control, Taiwan. 2009, pp.958-961.

[37] K. M. Hung, C.T. Hsieh: 'A Real-Time Mobile Vehicle License Plate Detection and Recognition', Journal of Science and Engineering, 2010, vol. 13, pp.433-442.

[38] N. Otsu, 'A Threshold Selection Method for Gray Level Histograms', IEEE Transactions on System, Man and Cybernetics, 1979.

[39] S.S. Beauchemin, M.A. Bauer, T. Kowsari, C. Ji: 'Portable and Scalable Vision-Based Vehicular Instrumentation for the Analysis of Driver Intentionality', IEEE Trans. On Instrumentation and Measurement, September, 2011, vol. 61, no. 2, pp. 391-401. 